\title{
A Change in the Treatment of Abdominal Aortic Aneurysms
}

\author{
José Honório Palma, Abner Moreira Sampaio, Fausto Miranda, Claudia Maria Rodrigues Alves, \\ J osé Augusto de Marcondes Souza, Enio Buffolo
}

São Paulo, SP - Brazil

\begin{abstract}
Objective - One of the most exciting potential applications of percutaneous therapy is the treatment of abdominal aneurysms.

Methods - Of 230 patients treated with a self-expanding polyester-lined stent-graft for different aortic pathologies at our institution, we selected 80 abdominal aneurysm cases undergoing treatment (from May 1997 to December 2002). The stent was introduced through the femoral artery, in the hemodynamic laboratory, with the patient under general anesthesia, with systemic heparinization, and induced hypotension.
\end{abstract}

Results - The procedure was successful in 70 (92.9\%) cases; 10 patients with exclusion of abdominal aortic aneurysms were documented immediately within the hemodynamic room and 5 patients persisted with a residual leak. Two surgical conversions were necessary. Additional stent-grafts had to be inserted in $3(3.7 \%)$ cases. In the follow-up, $91.4 \%$ of patients were alive at a mean follow-up of 15.8 months.

Conclusion - We believe that stent-grafts are an important tool in improving the treatment of abdominal aneurysms, and this new policy may change the conventional medical management of these patients.

Key words: abdominal aneurysm, self-expanding stentgraft
Conventional treatment of abdominal aneurysms has high morbidity and mortality rates, because in most cases such aneurysms occur in elderly patients with comorbidities, such as ischemic heart disease, previous aortic pathologies, chronic obstructive pulmonary disease, or renal dysfunction ${ }^{1}$. Due to the operative risk in this population, traditional surgical treatment has a high rate of potential complications.

Because the natural history of these diseases is well known and they have a high potential for complications, surgical treatment is recommended when diagnosis is made or signs are present of eminent rupture or enlargement ${ }^{2}$. Despite great advances in diagnostic imaging and in the development of new surgical approaches, mortality rates can be as high as 50\%, especially when patients are operated on as urgent cases ${ }^{3}$.

The introduction of stent-grafts into clinical practice by Parodi et al in ${ }^{4}$ as an alternative treatment for abdominal aortic aneurysms led to new perspectives. Since then, many groups have reported successful experiences in different aneurysmatic anatomies and with different types of endoprostheses allowing reduced morbidity and mortality rates for select groups ${ }^{5-7}$.

The clinical use of polyester-covered stents was pioneered at our institution for the treatment of type B dissections ${ }^{8}$. Good results obtained in this phase encouraged the development of new prostheses and catheters that can be inserted through a peripheral artery to treat thoracic and abdominal aortic diseases.

In this study, we report on our results and the complications observed in a series of 80 patients with abdominal aneurysms treated using self-expanding endovascular prostheses.

\section{Methods}

From May 1996 to December 2002, 230 cases (descending thoracic aortic diseases and abdominal aortic aneurysms) were treated with endoprostheses at our institution. We selected 80 patients with abdominal aneurysms. Of these, 36 had good distal necks or good anchorage sites for 
the stent in the distal aorta, and in 44, both iliac arteries in the aneurysms were compromised. All the patients had complex clinical symptoms with associated diseases, such as moderate-severe chronic obstructive pulmonary disease (17 patients), systemic hypertension (57 patients), renal insufficiency or creatinine $>1.5$ (15 patients), diabetes (10 patients), and heart failure or coronary disease (27 patients).

Diagnostic confirmations as well as measurement of the diameters and lengths for the prostheses were based on the following 2 tests: aortography and CT scan. Fifty patients had aneurysm diameters between 5.0 and $8.0 \mathrm{~cm}$. The institution's ethics committee approved the study protocol.

Anatomic criteria required for stenting were (1) good proximal landing zone of up to $32 \mathrm{~mm}$ near the renal artery and (2) iliac-femoral system compatible with a 20-Fr. device, without major bindings or obstructions.

The aortic stent-grafts are self-expanding polyesterlined stainless steel stents manufactured by Braile Biomédica, São José do Rio Preto, São Paulo-Brazil. These cylinders are highly resistant to radial collapse and maintain an ability to return to their original diameter. The prostheses have 3 parts implanted using 3 different catheters. The principal section is similar to a pair of shorts but has 1 leg shorter than the other. The other 2 parts are similar. The endoprostheses were carefully compressed and inserted in one 20 (principal) and two 17 (legs) Fr. (legs) catheters. Length and diameter are according to the dimensions of the diseased segment to be treated. Two different length options are available for the legs of the endoprostheses, 8.0 and $10.0 \mathrm{~cm}$, and after full expansion, diameters range from 2.0 to $3.4 \mathrm{~cm}$.

The procedures were performed in the catheter laboratory with the patient under general anesthesia, orotracheal intubation, and vital sign monitoring. A new arteriography was performed with 2 views (posteroanterior and left anterior oblique) using, when possible, calibrated catheters to confirm preoperative measurements. After aortography we chose the femoral artery, which is less tortuous for the introduction of the catheter with the stent, which was surgically dissected and opened with a transverse incision. A dose of $5000 \mathrm{U}$ of heparin was used for anticoagulation. Under radioscopic visualization, the catheter with the stent, using an extrastiff guidewire, was inserted into the femoral artery and slowly advanced towards the chosen site in the thoracic aorta.

The exact release site was marked by a radiopaque structure (for example: a vertebra or ruler placed on the patient's back before the procedure was started). First, the main section of the lined endoprosthesis was deployed into the renal artery where it self-expanded inside the aneurysm. The second step was to introduce the 2 "stent legs" or "stent branches" one at a time, approaching from the left and right femoral arteries. To confirm the effectiveness of the procedure, a new angiography was carried out a few minutes after the implantation of the stent-graft. After the procedure, the patients were removed to the postoperative recovery unit, where they were kept under observation for 24 hours. The patients were discharged after a control CT scan was performed (fig. 1).

\section{Results}

About half of the patients were $>70$ years of age. The mean age of the group was 71.5 years $(55-89$ years $)$ and $73 \%$ were male. All the patients treated by this method were serially followed up and in addition to the arteriography carried out during the procedure, CT scans and/or echocardiograms were performed to document the results and complications during hospitalization. The follow-up period ranged from 1 to 69 months with a mean of 15.8 months.

The procedure was technically successful in 70 patients (92.9\%) for the exclusion of aortic aneurysms (fig. 2). In 10 patients, a persistent or severe leak was detected, resulting in 1 emergency and 1 elective surgical conversion, resulting in a conversion rate of $2.5 \%$. For adequate repair, additional stent-grafts had to be inserted in 3 patients $(3.7 \%)$. Because of leakage, 5 patients remain under observation.

Six $(7.5 \%)$ in-hospital deaths occurred, and another 3 were related to the procedure. One patient developed multiple organ failure, one septicemia, and one pulmonary thromboembolism. The in-hospital survival rate was $92.5 \%$.

One $(1.4 \%)$ case of brachial thrombosis was observed in an individual whose access was achieved by using the Seldinger technique and who required surgical repair. Lower limb ischemia was observed in $2.9 \%$ of the patients, and 1 patient required endarterectomy of the femoral artery after the procedure. Another patient had deep vein thrombosis. Other complications were mild and transient and included renal failure (15 patients, $21.4 \%$ ), surgical wound infection (2 patients, $2.9 \%$ ), and peritoneal dialysis catheter infection (1 patient, 1.4\%). Fever was commonly seen (15 patients, $21.4 \%$ ). In most patients, the fever was not apparently related to an infection and was considered an inflammatory reaction to the prostheses. These patients were treated with antiinflammatory drugs and the temperature subsided.

After hospital discharge, 4 patients $(5.6 \%, 4 / 71)$ died. Two patients had sudden deaths, and 2 deaths were due to causes unrelated to aortic dissection or the procedure (one from a car accident and another from hepatic failure). At a mean follow-up of 15.8 months, $91.4 \%$ of the patients were alive.

\section{Discussion}

The concept of aneurysm repair by percutaneous insertion of an endovascular prosthesis was first suggested by Dotter ${ }^{9}$ in 1969 ; however, Parodi ${ }^{4}$ successfully pioneered treatment in a series of patients with abdominal aortic aneurysms. These authors used balloon-expanded endovascular prostheses and proved that the procedure was feasible and had low risks. Dake et al ${ }^{5}$ showed for the first time- the feasibility of treating descending thoracic aortic aneurysms introducing a self-expanding endovascular prosthesis through the femoral artery.

Also encouraging are the mid-term follow-ups of patients in this series, showing mortality and surgical conversion rates comparable to those in published works ${ }^{10,11}$. The 2 sudden deaths observed in this series might be related to aortic disease, demonstrating that these patients require a close follow-up. 


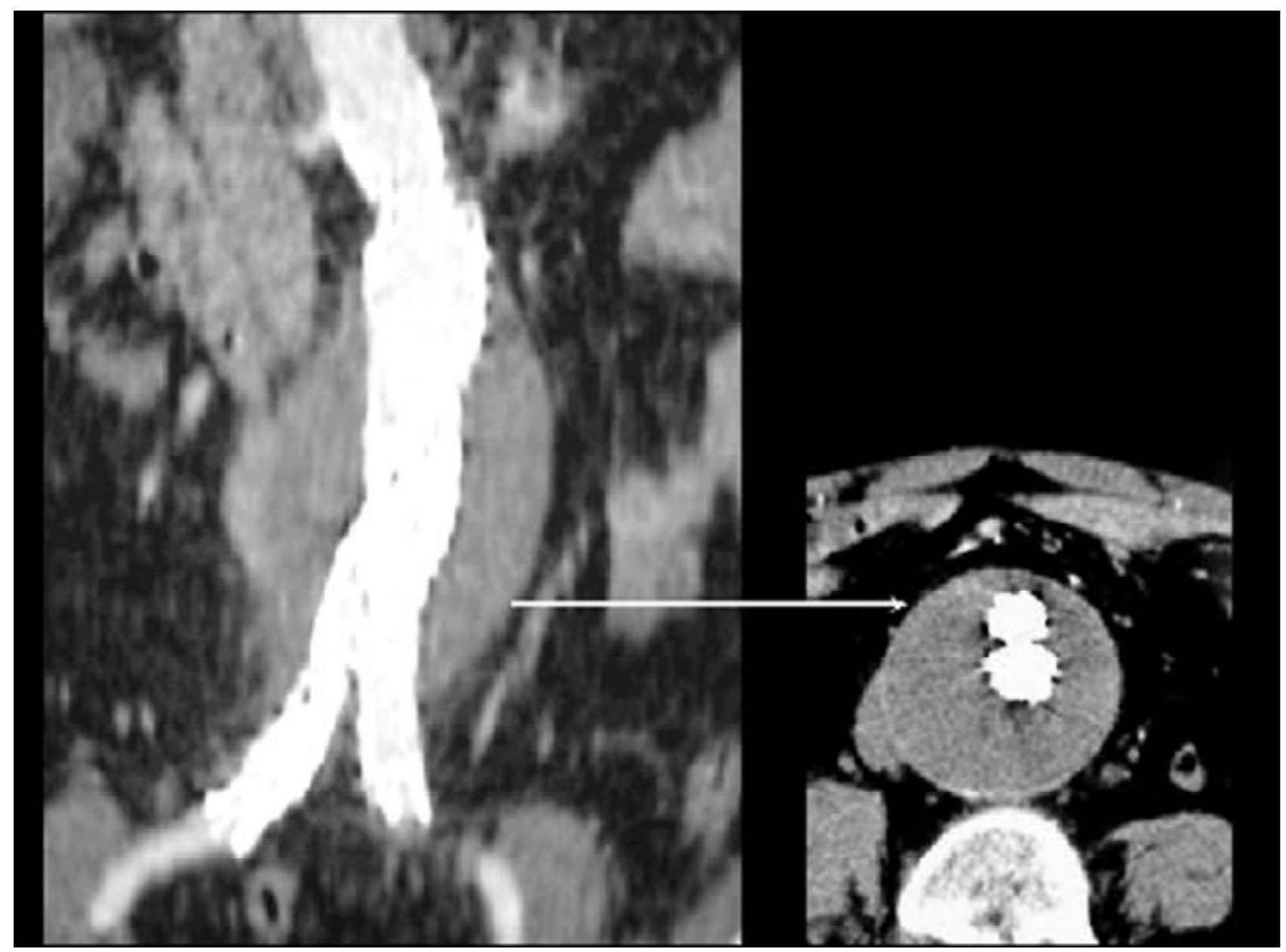

Fig. 1 - Abdominal CT scan with contrast showing the stent and an exclusion of the aneurysm.

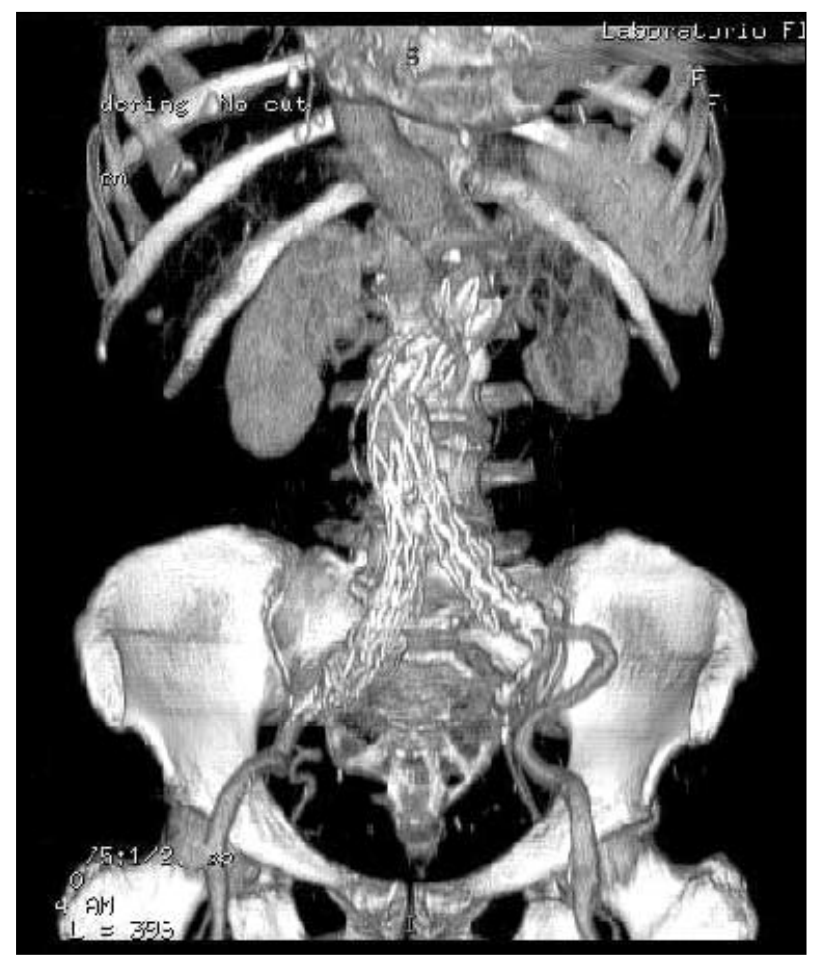

Fig. 2 - Bifurcated stent in the abdominal aorta.
It is worth noting that in this study no late ruptures occurred of the metallic structure of the endoprostheses nor did late migrations occur, and no cases were noted of rupture-expansion of the aneurysm or late leakage of the endoprostheses. The only late complication observed associated with the endoprosthesis was an occlusion of the left branch after 1 month.

The 4 patients who continue under observation for leakage have not experienced, until now, dilation of the aneurysmatic balloon, suggesting that some degree of protection is provided by the endoprosthesis.

The systemic complications in our study were few compared with those in to published investigations and considering the profile of our patients, who had an advanced mean age and thus were sufferers of co-morbidities.

We did not observe mortalities with elective surgical conversions, which suggests that we can be less restrictive in the indication of endovascular treatment because, when necessary, classical surgical treatment can be performed with the same traditional risks ${ }^{12}$.

An analysis of these data will perhaps allow us to conclude that we can treat abdominal aortic aneurysm patients who have aneurysms with smaller diameters and less tortuous vessels. Also, those patients can be treated who pre- 
sent with (a) an iliac-femoral system compatible with these catheters and (b) a proximal neck adequate for the anchoring of the prosthesis.

Other complications observed were considered minor. Fever not related to infection is frequent and may respond to nonhormonal antiinflammatory drug therapy. This commonly observed side effect may be an immune-mediated process, and the typical presentation is low-grade fever and mild leucocytosis. The use of antibiotics must be judiciously decided upon for this group of patients.
In conclusion, this successful initial experience in the treatment of severe cases with complications allows us to anticipate a promising future for this simple, less-invasive and low-risk approach. The follow-up of these cases shows it is possible to use the aortic stent-graft inserted in a catheter laboratory, with a low incidence of complications. However, a larger series, longer follow-up periods, and the development of prostheses and catheters (especially reducing the shaft size) that are more appropriate are required for this procedure to be more extensively used in the treatment of aortic disease.

\section{References}

1. Ashton HA, Buxton MJ, Day NE, et al. The Multicentre Aneurysm Screening Study (MASS) into the effect of abdominal aortic aneurysm screening on mortality in men a randomized controlled trial. Lancet 2002; 360: 1531-9.

2. Brown PM, Zelt DT, Sobolev B. The risk of rupture in untreated aneurysms: the impact of size, gender, and expansion rate. Vasc Surg 2003; 37: 280-4.

3. Beebe HG, Kritpracha B. Imaging of abdominal aortic aneurysm: current status. Ann Vasc Surg 2003: 24.

4. Parodi JC. Endovascular repair of abdominal aortic aneurysms and other arterial lesions. J Vasc Surg 1995; 21: 549-57.

5. Dake MD, Kato N, Michell RS. Endovascular stent-graft placement for the treatment of acute aortic dissection. N Engl J Med 1999; 340: 1546-52.

6. Nienaber CA, Fattori R, Lund G, et al. Nonsurgical reconstruction of thoracic aortic dissection by stent-graft placement. N Engl J Med 1999; 340: 1539-45.

7. Palma JH, Souza JAM, Alves CR, et al. Self-expandable aortic stent-grafts for treatment of descending aortic dissections. Ann Thorac Surg 2002; 73: 1138-45.
8. Palma JH, Almeida DR, Carvalho AC, et al. Surgical treatment of acute type B aortic dissection using an endoprosthesis (Elephant trunk). Ann Thorac Surg 1997; 63: 1081-4

9. Dotter CT. Transluminally-placed coilspring endoarterial tube grafts: long-term patency in canine popliteal artery. Invest Radiol 1969; 4: 329-32.

10. Schermerhorn ML, Finlayson SR, Fillinger MF, et al. Life expectancy after endovascular versus open abdominal aortic aneurysm repair: results of a decision analysis model on the basis of data from EUROSTAR. J Vasc Surg 2002; 36: 1112-20.

11. Matsumura JS, Brewster DC, Makaroun MS, et al. A multicenter controlled clinical trial of open versus endovascular treatment of abdominal aortic aneurysm. J Vasc Surg 2003; 37: 262-71.

12. Laheij RJ, van Marrewijk CJ, Buth J, Harris PL, EUROSTAR Collaborators. The influence of team experience on outcomes of endovascular stenting of abdomina aortic aneurysms. Eur J Vasc Endovasc Surg 2002; 24: 128-33. 